\title{
Interstitial Pneumonia Associated with Linear Immunoglobulin A/ Immunoglobulin G Bullous Dermatosis
}

\author{
Tomoyuki Kakugawa $^{\mathrm{a}}$ Saori Tomimura ${ }^{\mathrm{b}}$ Tomayoshi Hayashi $^{\mathrm{c}}$ \\ Noriho Sakamoto $^{a}$ Yuji Ishimatsu $^{\text {a Hiroshi Mukae }}{ }^{d}$ Shigeru Kohno $^{\text {a }}$ \\ a Second Department of Internal Medicine and b Department of Dermatology, Nagasaki University School of \\ Medicine, 'Department of Pathology, Nagasaki University Hospital, Nagasaki, and d Department of Respiratory \\ Medicine, School of Medicine, University of Occupational and Environmental Health, Kitakyushu, Japan
}

\section{Established Facts}

- The presence of immunoglobulin A (IgA) and IgG antibodies bound to the basement membrane zone of skin lesions is accepted as a characteristic feature of linear IgA/IgG bullous dermatosis.

- Lung disorders associated with linear IgA/IgG bullous dermatosis are extremely rare, and interstitial pneumonia associated with this dermatosis has not been previously reported.

\section{Novel Insights}

- We describe the first case of linear IgA/IgG bullous dermatosis-associated interstitial pneumonia in which direct immunofluorescence was used to demonstrate the deposition of IgA, IgG and C3 along the epithelial cell membrane and basement membrane of the bronchioles and alveoli.

- Similarly to in the skin, certain components in the epithelial cell membrane and basement membrane of the bronchioles and alveoli may be targeted by autoantibodies; this might contribute to the progression of interstitial pneumonia.

\section{Key Words}

Lung disorders · Linear immunoglobulin

A/immunoglobulin $\mathrm{G}$ bullous dermatosis .

Interstitial pneumonia

\begin{abstract}
A 76-year-old man with interstitial lung disease was admitted to our institution after developing persistent dyspnea upon effort. He also had a relapse of bullous eruptions on the skin of the trunk and extremities, previously diagnosed as
\end{abstract}

\section{KARGER}

E-Mail karger@karger.com www.karger.com/res
(อ) 2013 S. Karger AG, Base

0025-7931/13/0864-0347\$38.00/0
Tomoyuki Kakugawa, MD, PhD

Second Department of Internal Medicine Nagasaki University School of Medicine

1-7-1 Sakamoto, Nagasaki, 852-8501 (Japan)

E-Mail kakugawa@ nagasaki-u.ac.jp 
vesicular pemphigoid. Direct immunofluorescence of a skin biopsy specimen using fluorescence microscopy showed the linear deposition of immunglobulin $A(\lg A), \lg G$ and $C 3$ along the basement membrane. These findings indicated a definitive diagnosis of linear $\lg \mathrm{A} / \mathrm{lgG}$ bullous dermatosis. Chest computed tomography, bronchoalveolar lavage and transbronchial lung biopsy findings suggested nonspecific interstitial pneumonia. Direct immunofluorescence of the lung biopsy specimens using fluorescence microscopy also showed a deposition of $\lg \mathrm{A}$, IgG and C3 along the epithelial cell membranes and basement membranes of the bronchioles and alveoli. Lung disorders associated with linear $\lg \mathrm{A} /$ IgG bullous dermatosis are extremely rare and, to our knowledge, this is the first report of such a case of interstitial pneumonia.

Copyright $\odot 2013$ S. Karger AG, Basel

\section{Introduction}

Linear immunoglobulin A (IgA) bullous dermatosis (LABD) is an autoimmune subepidermal bullous disease characterized by linear IgA deposits in the epidermal basement membrane zone [1]. Some cases demonstrate linear deposits of IgG and IgA, i.e. linear IgA/IgG bullous dermatosis (LAGBD) [2]. Lung disorders associated with LABD or LAGBD are extremely rare. Here we describe the first case, to our knowledge, of interstitial pneumonia associated with LAGBD.

\section{Case Report}

A 76-year-old man with interstitial lung disease was referred to our institution after developing persistent dyspnea upon effort of grade 2 based on the modified Medical Research Council Dyspnea Scale [3]. He denied having any cough. He was an exsmoker (20 cigarettes/day for 51 years), having quit 6 years previously. He had been diagnosed with vesicular pemphigoid 21 years previously, and had been stable on a maintenance dose of oral prednisolone of $8 \mathrm{mg} /$ day. However, his skin lesions had relapsed at the time of his referral. He had been taking the following medications other than prednisolone: zolpidem tartrate, alendronate sodium hydrate, famotidine, ketotifen fumarate, telmisartan, furosemide, torasemide and allopurinol. These were all prescribed by his primary care physician and had not been changed for several years. He had never been exposed to any infectious, toxic or environmental factor that could cause interstitial lung disease. Physical examination demonstrated multiple pustules, vesicles and erosions with erythema on the trunk and proximal upper and lower extremities (fig. 1). He was afebrile. On chest auscultation, fine crackles were detected bilaterally. No extrathoracic signs were detected besides the skin lesions. Arterial blood gas analysis on room air revealed a $\mathrm{pH}$ of $7.48, \mathrm{PaO}_{2}$ of $77 \mathrm{~mm} \mathrm{Hg}$ and $\mathrm{PaCO}_{2}$ of $32 \mathrm{~mm} \mathrm{Hg}$. Laboratory examinations upon admission revealed a white blood cell count of $10,600 / \mathrm{mm}^{3}$ with $66 \%$ neutrophils. Anti-single strand deoxyribonucleic acid antibody was positive $(27.8 \mathrm{AU} / \mathrm{ml})$, but other autoantibodies to specific antigens, including anti-nuclear antigen, were all negative. Pulmonary function tests demonstrated the following diffusion impairment: \% predicted vital capacity (\%VC) $83.3 \%$, \% predicted forced vital capacity (\%FVC) $84.0 \%$ and $\%$ predicted diffusing capacity for carbon monoxide (\%DLCO) $64.5 \%$. A biopsy specimen from a skin lesion showed subepidermal blisters with lymphocyte infiltrates in the dermis and inside the blister cavity. Direct immunofluorescence of the specimen showed the linear deposition of IgG, IgA and C3 along the basement membrane. These findings indicated a definitive diagnosis of LAGBD. Chest radiography showed reticular shadowing with basilar predominance (fig. 2). Chest computed tomography (CT) showed patchy ground-glass attenuation and reticular opacities, predominantly in the peripheral lung fields (fig. 3). Although chest CT revealed traction bronchiectasis in the bilateral lower lobes, neither honeycombing nor mediastinal lymphadenopathy were evident. Bronchoscopy revealed no abnormalities from the pharynx to the subsegmental bronchi. Examination of bronchoalveolar lavage fluid from the left $\mathrm{B} 5$ bronchus showed a total cell count of $1.18 \times 10^{5}$ cells $/ \mathrm{ml}$, comprising $65 \%$ macrophages, $30 \%$ lymphocytes, $2 \%$ neutrophils and $3 \%$ eosinophils. The CD4/CD8 ratio of lymphocyte subsets was 1.42 . Siderophages were not evident. Transbronchial lung biopsy specimens of the left lateral basal segment (S9) showed alveolar septal thickening and inflammatory cell infiltration (fig. 4). Direct immunofluorescence of the specimen revealed deposition of IgA, IgG and C3 along the epithelial cell membrane and basement membrane of the bronchioles and alveoli (fig. 5). Although bronchoalveolar lavage fluid findings, imaging findings and histopathological analysis of the transbronchial lung biopsy specimens suggested a pattern of nonspecific interstitial pneumonia, it was difficult to make a definite pathological diagnosis because a surgical lung biopsy was not performed. Oral prednisolone was increased to $20 \mathrm{mg} / \mathrm{day}$, which was then tapered off gradually to a maintenance dose of $13 \mathrm{mg} /$ day. Although the skin lesions improved, the pulmonary lesions did not. The patient's dyspnea upon effort and the chest CT findings remained stable over 18 months of follow-up. However, pulmonary function tests demonstrated a gradual deterioration, and the results 18 months after presentation were as follows: \%VC 74.2\%, \%FVC 73.2\% and \%DLCO 32.5\%.

\section{Discussion}

To our knowledge, this is the first reported case of interstitial pneumonia associated with LAGBD. Both LABD and LAGBD may present with lesions of the skin or mucous membranes, or both. Any mucosal surface may be affected, including the oral cavity, conjunctiva, nose, genitalia, pharynx, larynx, anus and esophagus $[4$, 5]. Serious adverse consequences of cicatrizing mucosal disease include airway obstruction in the pharynx and
Kakugawa/Tomimura/Hayashi/ Sakamoto/Ishimatsu/Mukae/Kohno 
Fig. 1. A photograph of the skin lesions on the trunk and extremities shows tense blisters, erosions and erythema with some crusts.
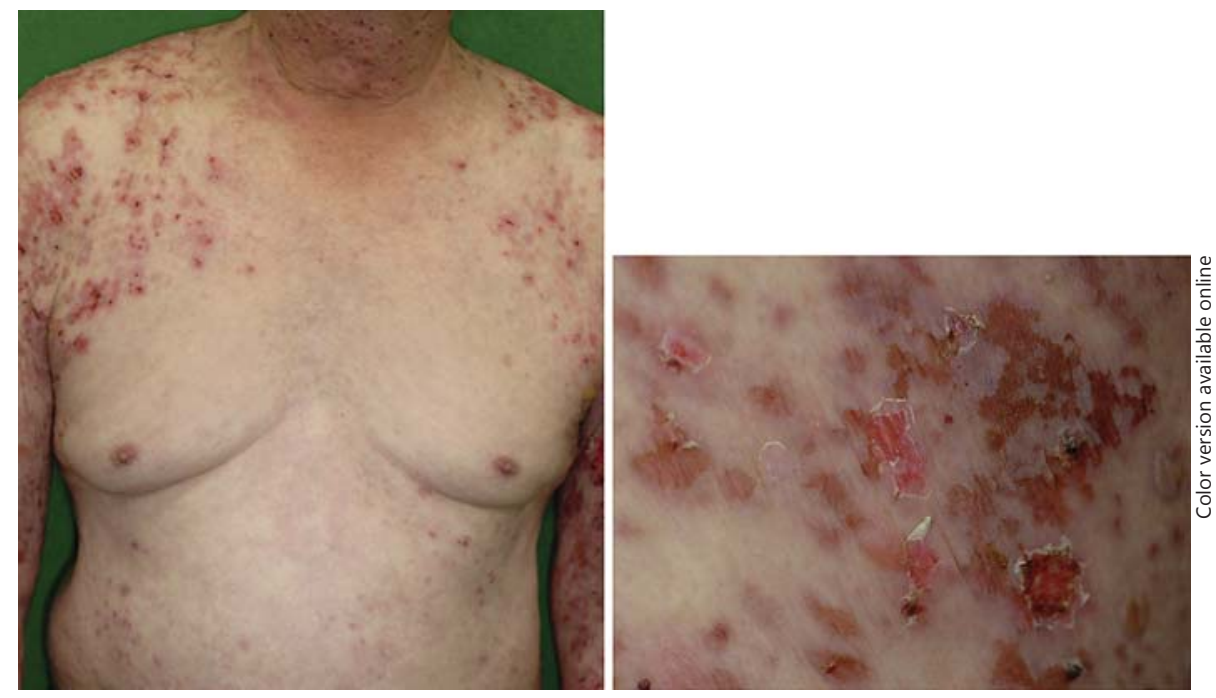

larynx $[5,6]$. LABD or LAGBD rarely involves the lower respiratory tract. The few case reports of respiratory lesions associated with these skin conditions have described involvement of the bronchial mucosa with subepithelial blister formation [7], sarcoidosis [8] and tracheomalacia [5, 9]. However, in this case, bronchoscopy revealed no abnormalities from the pharynx to the subsegmental bronchi. To the best of our knowledge, interstitial pneumonia associated with LAGBD has not been reported previously.

Although the presence of IgA and IgG antibodies bound to the basement membrane zone is considered a characteristic feature of LAGBD, the mechanism of lesion formation is not well understood. Tissue injury resulting from an antibody-induced local inflammatory response and the release of proteolytic enzymes by neutrophils and other inflammatory cells may contribute to the development of skin and mucosal lesions [4]. Most patients with LABD have IgA 1 antibodies that target a $97-\mathrm{kDa}$ antigen and a $120-\mathrm{kDa}$ antigen within the basement membrane zone. Both of these antigens are fragments of the extracellular portion of BP180, a transmembrane protein with a key role in epidermal-dermal adhesion $[10,11]$. Other reported autoimmune targets for LABD include collagen VII, BP230 and LABD97 [2].

Abnormalities in epithelial cell function have been proposed as central to the pathogenesis of interstitial pneumonia. In this case, direct immunofluorescence of the biopsied lung specimen showed deposition of IgA, IgG and C3 along the epithelial cell membrane and base-

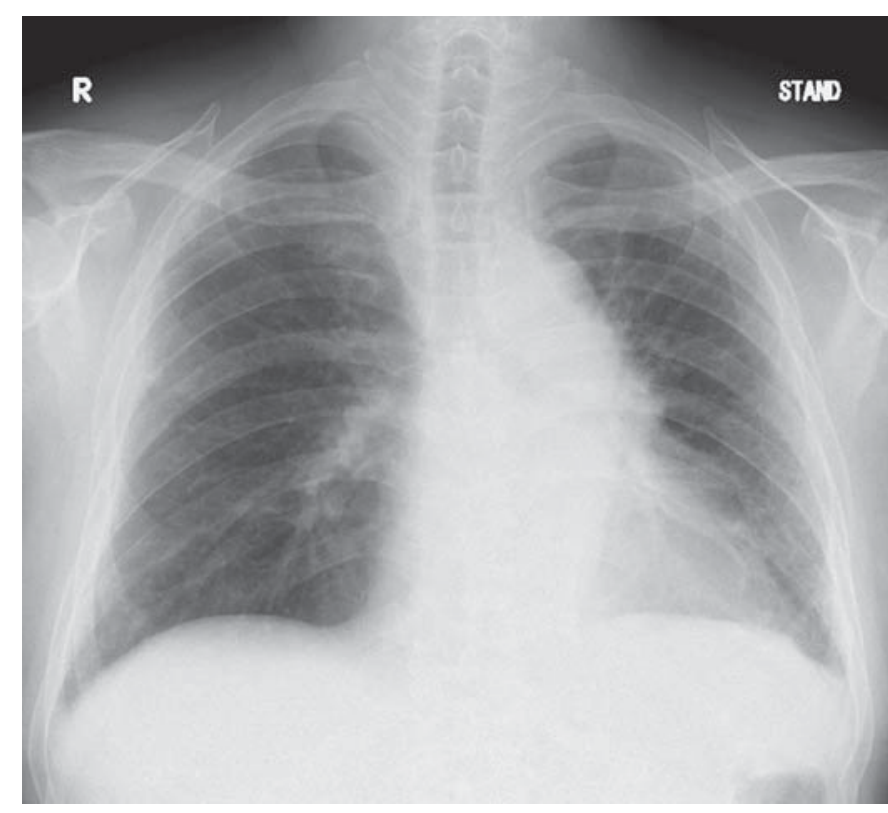

Fig. 2. Chest radiography shows reticular shadowing with basilar predominance.

ment membrane of the bronchioles and alveoli. Similarly to in the skin, certain components in the epithelial cell membrane and basement membrane of the bronchioles and alveoli may be targeted by autoantibodies. Yoshioka et al. [12] reported similar findings in a patient with bullous pemphigoid. This immunological reaction might 


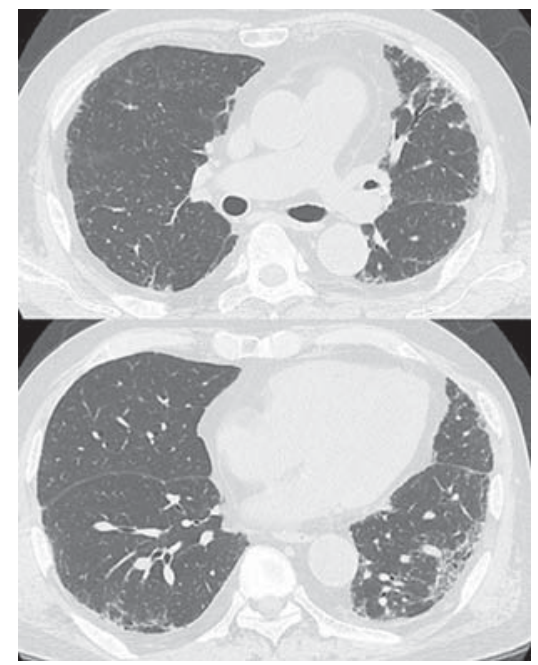

Fig. 3. Chest CT shows patchy ground-glass attenuation and reticular opacities, predominantly in the peripheral lung fields.

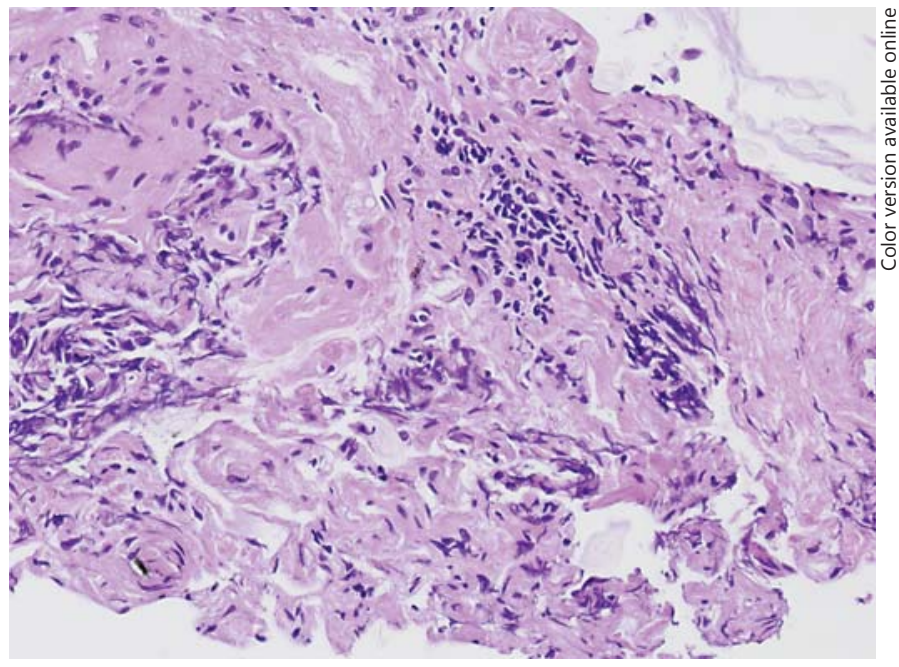

Fig. 4. Transbronchial lung biopsy specimens of the left lateral basal segment (S9) show alveolar septal thickening and inflammatory cell infiltration. HE. $\times 400$.
Fig. 5. a-f Sequentially sectioned pairs. $\times 400$. Direct immunofluorescence of the biopsied lung specimen using a fluorescence microscope shows deposition of IgA (FITC-labeled antibody anti-IgA) (a), IgG (FITC-labeled antibody anti-IgG) (c) and C3 (FITC-labeled antibody anti-C3) (e) along the epithelial cell membrane and basement membrane of the bronchioles. Negative control studies using anti-IgA antibody without FITC (b), anti-IgG antibody without FITC (d) and phosphatebuffered saline (f) revealed no significant positive findings.
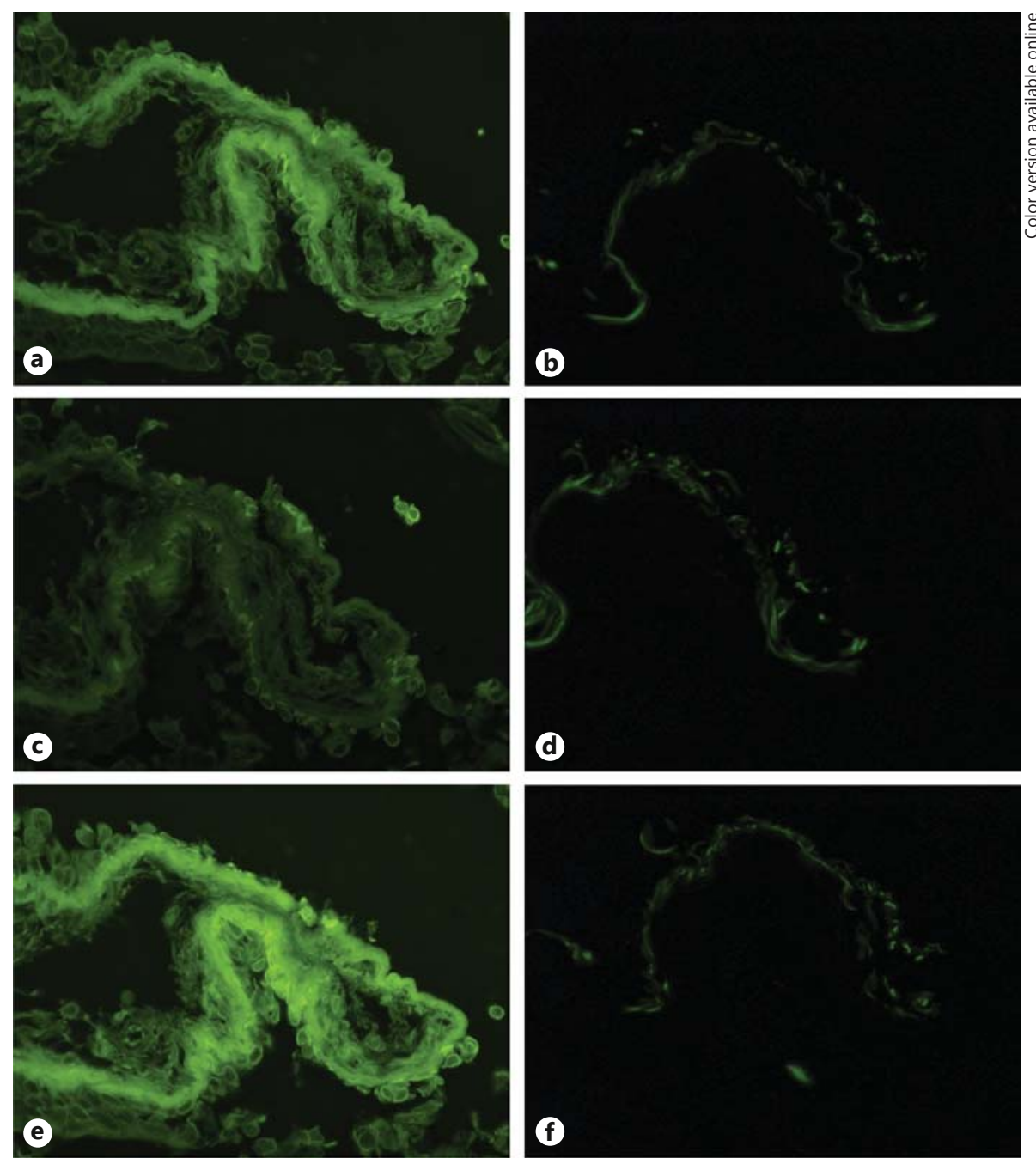
have contributed to the progression of interstitial pneumonia in our patient.

In conclusion, we described the first case of LAGBDassociated interstitial pneumonia. Clinicians should be aware that interstitial pneumonia may be a pulmonary manifestation of LAGBD.

\section{Financial Disclosure and Conflicts of Interest}

The authors do not have any competing interests and/or bias with regard to the publication.

\section{References}

$>1$ Zone JJ: Clinical spectrum, pathogenesis and treatment of linear IgA bullous dermatosis. J Dermatol 2001;28:651-653.

$\checkmark 2$ Zone JJ, Pazderka Smith E, Powell D, Taylor TB, Smith JB, Meyer LJ: Antigenic specificity of antibodies from patients with linear basement membrane deposition of IgA. Dermatology 1994;189(suppl 1):64-66.

$>3$ Fletcher CM, Elmes PC, Fairbairn AS, Wood $\mathrm{CH}$ : The significance of respiratory symptoms and the diagnosis of chronic bronchitis in a working population. Br Med J 1959;2: 257-266.

4 Fortuna G, Marinkovich MP: Linear immunoglobulin A bullous dermatosis. Clin Dermatol 2012;30:38-50.
5 Gluth MB, Witman PM, Thompson DM: Upper aerodigestive tract complications in a neonate with linear IgA bullous dermatosis. Int J Pediatr Otorhinolaryngol 2004;68:965-970.

6 Sato K, Hanazawa H, Sato Y, Watanabe J: Initial presentation and fatal complications of linear IgA bullous dermatosis in the larynx and pharynx. J Laryngol Otol 2005;119:314318.

7 Verhelst F, Demedts M, Verschakelen J, Verbeken E, Marien K, Peeters C: Adult linear IgA bullous dermatosis with bronchial involvement. Br J Dermatol 1987;116:587-590.
-8 Porter WM, Hardman CM, Leonard JN, Fry L: Sarcoidosis in a patient with linear IgA disease. Clin Exp Dermatol 1999;24:67-70.

-9 Hruza LL, Mallory SB, Fitzgibbons J, Mallory GB Jr: Linear IgA bullous dermatosis in a neonate. Pediatr Dermatol 1993;10:171-176.

10 Guide SV, Marinkovich MP: Linear IgA bullous dermatosis. Clin Dermatol 2001;19:719727.

11 Chorzelski TP, Jablonska S, Maciejowska E: Linear IgA bullous dermatosis of adults. Clin Dermatol 1991;9:383-392.

12 Yoshioka D, Ishii H, Uchida T, Fujiwara S, Umeki K, Sakamoto N, Kadota J: Interstitial pneumonia associated with bullous pemphigoid. Chest 2012;141:795-797. 